\title{
Role of the Basolateral Nucleus of the Amygdala in the Formation of a Phobia
}

\author{
Sandra E. File, B.Sc., Ph.D., D.Sc., Luis E. Gonzalez, M.D., Ph.D., and Rachael Gallant, B. Sc.
}

Specific phobia is an anxiety disorder that does not respond to benzodiazepines. The elevated plus-maze test of anxiety is sensitive to benzodiazepines on trial 1, but during the first 5-min trial the nature of the anxiety generated changes to a specific fear of heights, and, as a result, on trial 2 the rats no longer respond to benzodiazepines. However, rats that received reversible bilateral lesions of the basolateral amygdala (by lidocaine injection) immediately after trial 1 responded with an anxiolytic response to chlordiazepoxide when tested $48 \mathrm{~h}$ later on trial 2. Those that received vehicle injections after trial 1 showed the usual lack of response to chlordiazepoxide on trial 2. Thus, the basolateral amygdala plays a crucial role in the consolidation of information that leads to the formation of a specific phobia and subsequent insensitivity to benzodiazepines.

[Neuropsychopharmacology 19:397-405, 1998]

(C) 1998 American College of Neuropsychopharmacology. Published by Elsevier Science Inc.
KEY WORDS: Anxiety; Phobia; Amygdala; Benzodiazepines; Emotional memory; Plus-maze

Several different anxiety disorders are clinically recognized, each with differing sensitivity to benzodiazepine treatment. Thus; whereas the benzodiazepines are most effective in generalized anxiety disorder, they are less effective in panic disorder (where antidepressant therapy is the treatment of choice), and not at all effective against specific phobias, for which exposure therapy is most effective (Marks 1987; Tyrer 1989). There are also several different animal tests of anxiety, and principal component analysis has confirmed that the measures derived from different tests load on independent factors and thus are reflecting quite distinct states of anxiety (File 1991). There is also growing evidence that different brain regions and neurotransmitter systems modulate different measures of fear and anxiety (Pesold and Treit 1994, 1995; File and Gonzalez 1996;

From the Psychopharmacology Research Unit, UMDS, Guy's Hospital, London, UK.

Address correspondence to: Professor S.E. File, Psychopharmacology Research Unit, UMDS, Guy's Hospital, London SE1 9RT, UK

Received November 13, 1997; revised February 16, 1998; accepted February 16, 1998.
Gonzalez and File 1997; Killcross et al. 1997), which accords with the distinct drug treatments used clinically for different anxiety disorders.

The elevated plus-maze is a well-validated (Pellow et al. 1985) and widely used test of anxiety in which a rat or mouse is faced with a choice of open elevated arms or those enclosed by a high wall. The animal is placed in an approach-avoidance conflict situation in which exploration of the novel open arms is pitted against avoidance generated by fear. Treit et al. (1993) found that on the first exposure to the plus-maze, it is the open aspect of the arm, rather than its elevation, that is the main anxiogenic stimulus. On trial 1, this test is sensitive to the effects of benzodiazepines, which increase the percentage of time spent on the open arms and the percentage of entries onto open arms, in mice or rats exposed for the first time to the maze. However, it soon became clear that if rats or mice were replaced on the plus-maze for a second 5 min trial, they became insensitive to the anxiolytic effects of benzodiazepines or barbiturates (File 1990; File 1993; Rodgers et al. 1992; Rodgers and Shepherd 1993). The drug state of the animal on trial 1 is unimportant to this insensitivity to benzodiazepines on trial 2, and the interval between trials can be from $24 \mathrm{~h}$ to 2 weeks; the crucial factor is experi- 
ence of the open arms (File et al. 1990), which must include exploration of and head-dipping over the edges of the arm and, hence, knowledge of the drop (Fernandes and File 1996). The acquisition of fear of heights is probably based more on tactile than visual cues, because the rat is myopic and with an undifferentiated floor would be unable to make graded depth judgments; it is also likely that depth is judged in an "all-or-none" manner-low enough and safe to jump versus high enough to evoke fear. Thus, while the state of fear or anxiety generated by initial exposure to the plus-maze is unconditioned, by trial 2 it has been replaced by a different form of fear, which was rapidly acquired during the trial 1 experience. Because of the insensitivity to benzodiazepines on trial 2 and the importance of the fear of height on this trial, it was proposed that the nature of anxiety evoked was similar to a simple, or specific phobia (File and Zangrossi 1993; File et al. 1996); as such, this rapidly acquired fear is likely to be based on an innate preparedness, and its insensitivity to benzodiazepine treatment suggests it is different from other forms of conditioned fear. Principal component analyses confirmed that the measures of anxiety for trials 1 and 2 load on two independent factors (File et al. 1993; Rodgers and Johnson 1995; Fernandes and File 1996) and, thus, reflect two different states of anxiety.

The amygdaloid complex has long been implicated in anxiety disorders and conditioned fear (Davis 1992; LeDoux 1996; McGaugh 1995; Everitt and Robbins 1992). Recent experiments have elucidated distinctive roles for different amygdaloid nuclei with, for example, the central nucleus playing a key role in reflexive, automatic and Pavlovian conditioned responses to aversive stimuli and the basolateral nucleus playing a key role in voluntary or choice behavior based on emotional events (Killcross et al. 1997). The basolateral nucleus would, therefore, seem an ideal candidate for a mediating role in the formation of the phobic response that seems to be acquired during the first few minutes of exposure to the plus-maze and is demonstrated by an insensitivity to benzodiazepines on a subsequent exposure to the maze. McGaugh et al. (1996) proposed that the prime role of the basolateral nucleus of the amygdala is to regulate the consolidation or storage, in other brain areas, of information with conditioned motivational value. Therefore, it plays a crucial role in the consolidation of emotional learning, but it is not necessarily the locus of storage of that information. Thus, whereas lesion of the basolateral nucleus before acquisition or retention testing attenuates performance, only a reversible lesion induced by lidocaine shortly after acquisition, entirely blocks retention of inhibitory avoidance conditioning (Parent and McGaugh 1994).

The purpose of the present study was to investigate the role of the basolateral nucleus of the amygdala in the consolidation of information derived from trial 1 in the plus-maze, which, in turn, leads to an insensitivity to benzodiazepines on trial 2 . Therefore, animals were given a single, 5-min undrugged exposure to the maze, followed by an intra-amygdaloid injection of lidocaine or vehicle. They were then tested again in the plus-maze 2 days later after IP injection with chlordiazepoxide or vehicle. Rats that had received the vehicle injection into the amygdala were expected to demonstrate the usual insensitivity to chlordiazepoxide on trial 2. In contrast, those in which the amygdala was inactivated by lidocaine would be unable to consolidate emotional information from trial 1 and would, thus, respond to chlordiazepoxide on trial 2 , because the formation of the specific phobia would have been prevented. Because only one dose of chlordiazepoxide was to be used on trial 2, we used a pilot group of rats to establish an effective anxiolytic, nonsedative dose on trial 1 . These rats were not used in the subsequent experiment. This precaution was taken because of seasonal and batch differences in sensitivity to the anxiolytic effects of benzodiazepines.

\section{MATERIALS AND METHODS}

\section{Animals and Surgery}

Thirty male hooded Lister rats (Harlan, Bicester, UK) were individually housed after surgery in a dimly lit room maintained at $22^{\circ} \mathrm{C}$, with lights on from 0700 to $1900 \mathrm{~h}$. Food and water were freely available. The rats weighed 250 to $300 \mathrm{~g}$ at the time of surgery.

Before surgery, the stereotaxic coordinates were verified histologically on two rats from the same batch. One week after arrival, animals were anesthetized by inhalation of 3\% halothane (May and Baker, UK) in oxygen and positioned in a stereotaxic frame (Kopf Instruments). The skull was exposed, and the incisor bar adjusted so that bregma and lambda were at the same height. Three indentations were made in the skull to accommodate screws that, together with the application of dental cement, held the cannulae in place. For bilateral cannulation of the basolateral nuclei of amygdala, 12 -mm long steel guide cannulae (23 gauge) were positioned $3.3 \mathrm{~mm}$ posterior to bregma, lateral $\pm 4.5 \mathrm{~mm}$, and vertical $-5.7 \mathrm{~mm}$, thus siting them $2 \mathrm{~mm}$ above the target area. Cannulae were kept patent using 12-mm long stainless steel stylets (30 gauge, Cooper's Needle Works Ltd, UK). To accustom the animals to handling and to keep the cannulae patent, each day following surgery the rats were gently wrapped in a cloth, and the stylets were replaced. The animals were allowed at least 5 days recovery from surgery before behavioral testing.

\section{Drugs and Microinfusion}

Lidocaine (2-Diethylamino-N-[2,6-dimethylphenyl]-acetamide hydrochloride, Sigma Chemical Co., Poole, Dor- 
set, UK) was dissolved in artificial cerebrospinal fluid (aCSF) of the following composition (mM); $\mathrm{NaCl}$ 126.6, $\mathrm{NaHCO}_{3} 27.4, \mathrm{KCl} 2.4, \mathrm{KH}_{2} \mathrm{PO}_{4} 0.5, \mathrm{CaCl}_{2} 0.89, \mathrm{MgCl}_{2}$ $0.8, \mathrm{Na}_{2} \mathrm{HPO}_{4}$ 0.48, and glucose 7.1, $\mathrm{pH}=7.4$. Immediately after the 1st day's test, each rat was removed from the apparatus and immediately received a bilateral infusion of either lidocaine $(40 \mu \mathrm{g} / \mu \mathrm{l})$ or aCSF, delivered at the rate of $0.2 \mu \mathrm{l} / \mathrm{min}$ for $1 \mathrm{~min}$, using a microdialysis pump (CMA/102). The injection needles (30 gauge) were inserted $2 \mathrm{~mm}$ below the tip of the cannulae and were left in place for a further $1 \mathrm{~min}$ to allow diffusion away from the tip. Chlordiazepoxide hydrochloride (Sigma) was dissolved in distilled water to a concentration of $1.25 \mathrm{mg} / \mathrm{ml}$. Rats received an IP injection of chlordiazepoxide $(2.5 \mathrm{mg} / \mathrm{kg})$ or an equal volume of distilled water, 30 min before testing on trial 2 .

\section{Apparatus}

The plus-maze was made of wood and consisted of two opposite open arms $50 \times 10 \mathrm{~cm}$ and two opposite arms enclosed by $40-\mathrm{cm}$ high walls. The arms were connected by a central $10 \times 10 \mathrm{~cm}$ square, thus the maze formed a "plus" shape. The maze was elevated $50 \mathrm{~cm}$ from the floor and lit by dim light. A closed-circuit TV camera was mounted vertically over the maze and the behavior was scored from a monitor in an adjacent room. The numbers of entries onto open and enclosed arms and the times spent on the open and closed arms were recorded. An arm entry was defined as being when all four paws entered the arm; exit from an arm was defined as being when the forepaws left that arm. The scores were entered directly into an IBM computer. A change in the percentage of time spent on the open arms is the best measure of a change in anxiety, and the number of closed arm entries is the best measure of general activity (File 1991).

\section{Procedure}

Rats were randomly allocated, half to receive intraamygdaloid injections of lidocaine, and half to receive aCSF. On the 1st test day, each rat was placed (without any drug treatment) in the central square of the plusmaze, and its behavior was scored for $5 \mathrm{~min}$. At the end of each trial, the maze was wiped clean with a damp cloth. Immediately after this trial, rats received intraamygdala injections of lidocaine or aCSF, as described above. Animals in both groups were then randomly allocated ( $n=7-8$ /group) to be tested on trial 2 with chlordiazepoxide or vehicle. These injections were given IP $30 \mathrm{~min}$ before trial 2 in the plus-maze. Trial 2 took place $48 \mathrm{~h}$ after trial 1 , and the rats were tested in an order randomized for previous amygdala injection and for drug treatment on the test day. The rats were scored as before, by an observer who was blind to their drug treatment. Testing on both days took place between 0800 and $1200 \mathrm{~h}$.

\section{Histology}

At the end of behavioral testing, all animals were sacrificed, the brains were removed, and the injection site was verified histologically (according to the atlas of Paxinos and Watson 1986) by a person blind to drug treatment. Frozen brains were sectioned on an British Optical Cryocut at $10 \mu \mathrm{m}$. Sections were obtained parallel to the stereotaxic planes by adjusting the angle of cutting. Sections were taken directly from the cryotome knife on coated slides (poly-L-lysine 10\%) with the help of an antiroll device.

To visualize the boundaries of the basolateral nuclei, the method for demonstration of AChE was used following the procedures described by Paxinos and Watson (1986). Slides with fresh, unfixed tissue were incubated for $15 \mathrm{~h}$ in the following solution: $100 \mathrm{ml}$ of stock solution to which had been added $116 \mathrm{mg}$ of S-acetylthiocholine iodide and $3.0 \mathrm{mg}$ ethopropazine (May and Baker). Subsequently, the slides were rinsed with water and developed for $10 \mathrm{~min}$ in $1 \%$ sodium sulphide $(1.0 \mathrm{~g}$ in $100 \mathrm{ml}$ of $\mathrm{H}_{2} \mathrm{O}$ ) at $\mathrm{pH}$ 7.5. They were then rinsed with water and immersed in $4 \%$ paraformaldehyde in phosphate buffer for $8 \mathrm{~h}$ and then allowed to dry. Finally they were cover-slipped with DPX (Raymond A. Lamb, London). The stock solution was $50 \mathrm{mM}$ sodium acetate buffer at $\mathrm{pH} 5$, which was made by adding $6.8 \mathrm{~g}$ of sodium acetate, $1.0 \mathrm{~g}$ of copper sulphate crystals, and 1.2 $\mathrm{g}$ of glycine to $1.0 \mathrm{l}$ of $\mathrm{H}_{2} \mathrm{O}$ and lowering the $\mathrm{pH}$ to 5.0 with $\mathrm{HCl}$.

\section{Statistics}

Because the measures derived from the plus-maze were normally distributed, and there was homogeneity of variance between the groups, the data were analyzed by analysis of variance (ANOVA). The data for trial 2 were analyzed by a two-way ANOVA with amygdala injection after trial 1 and drug treatment on trial 2 as the two factors. The data for the rats tested undrugged on both trials were analyzed by a 2-factor between-within ANOVA, with amygdala injection after trial 1 as the between-group factor and trials as the within-group factor. After ANOVA, comparisons between individual groups were then made with Duncan's tests, and the significances of these are shown in the figures.

\section{RESULTS}

\section{Histology}

Figure 1, depicting coronal slices through the amygdala complex shows the positions of injection sites within 
the basolateral nucleus of the amygdala (black dots) and the positions of the three placements (two bilateral and one unilateral) falling outside the target area (shown by crosses). Data for these animals were excluded from statistical analysis, which left $n=6$ to 7 / group. Figure 1 also shows a coronal section stained for acetylcholinesterase to visualize the borders of the basolateral nucleus of the amygdala, with a typical bilateral cannulation to demonstrate the extent of mechanical damage.

\section{Trial 2 in the Plus-Maze}

All the rats were exposed undrugged to the plus-maze on trial 1. It can be seen from Figure 2 that the animals that received aCSF injections into the basolateral nucleus of the amygdala immediately after trial 1 showed the usual insensitivity to chlordiazepoxide on trial 2 in the plus-maze. In contrast to the usual pattern observed on trial 2, the rats that received a lidocaine infusion immediately after trial 1 showed a significant anxiolytic response to chlordiazepoxide, as indicated by an increased percentage of time spent on the open arms and an increased percentage of entries onto the open arms (see Figure 2). This reflected a specific anxiolytic response, because chlordiazepoxide did not affect the number of closed arm entries (mean \pm SEM: control $=$ $10.8 \pm 3.0$, chlordiazepoxide $=9.0 \pm 1.6$ ). The lack of response to chlordiazepoxide in the group receiving aCSF after trial 1 cannot be attributed to locomotor
A

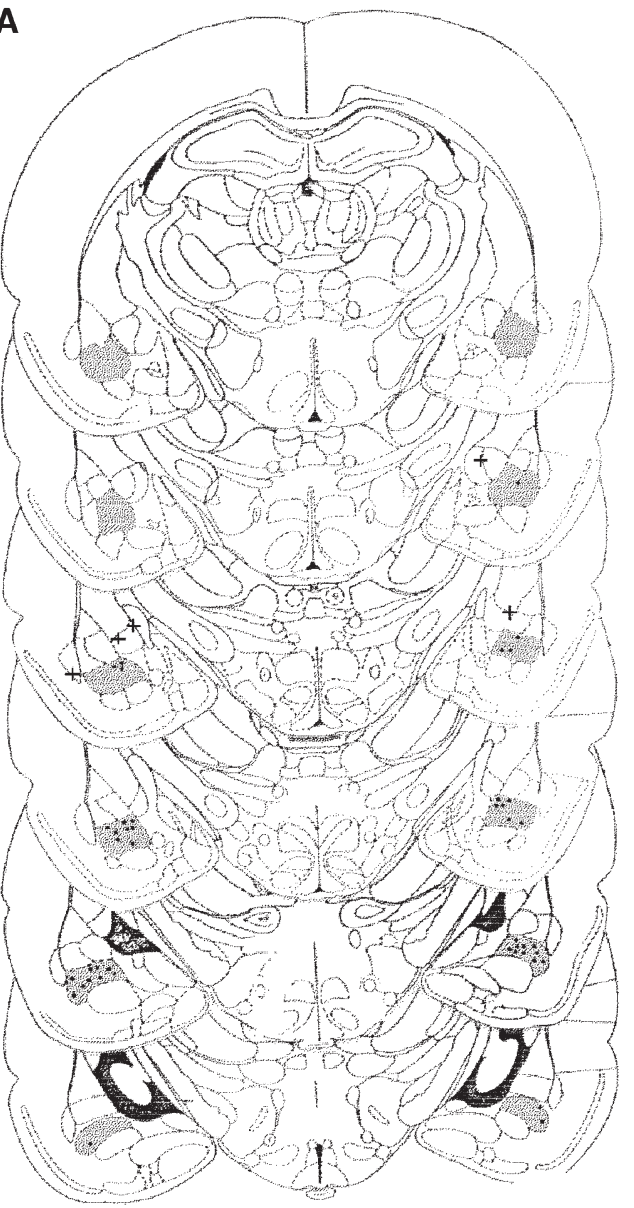

B

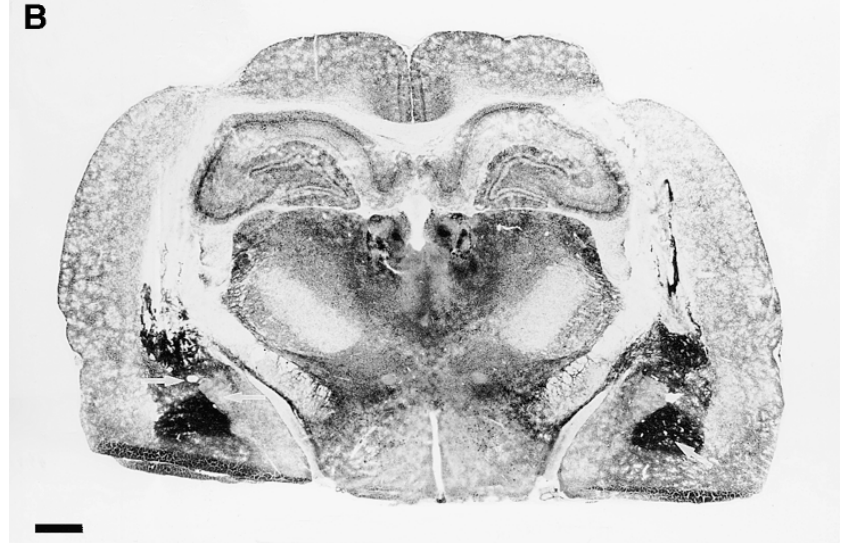

$-3.30$

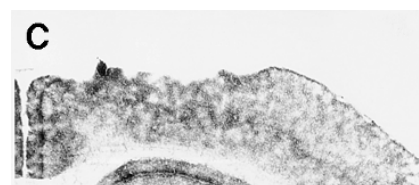

$-3.60$

$-3.80$

Figure 1. (A) Diagrammatic representation of coronal sections (2.56-3.80 $\mathrm{mm}$ posterior to bregma) through the rat brain showing the target area (shaded) of the basolateral amygdala nucleus. Placements falling inside this area are shown by filled circles; those falling outside (two bilateral and one unilateral placement) are shown by crosses. These mark the site of the tip of the injection needle. (B) Coronal section (approximately $3.14 \mathrm{~mm}$ posterior to bregma) stained for acetylcholinesterase, showing the basolateral nucleus of the amygdala (dark areas at the bottom as marked by arrow on right side), clearly distinguished from the central nucleus (lying above and medially as marked by arrow on left side). Also shown are the injection sites for placements falling outside the target area; graticule $1 \mathrm{~mm}$. (C) Coronal section (approximately 3.30 posterior to Bregma) showing typical injection site into the basolateral nucleus of the amygdala; graticule $0.5 \mathrm{~mm}$. 

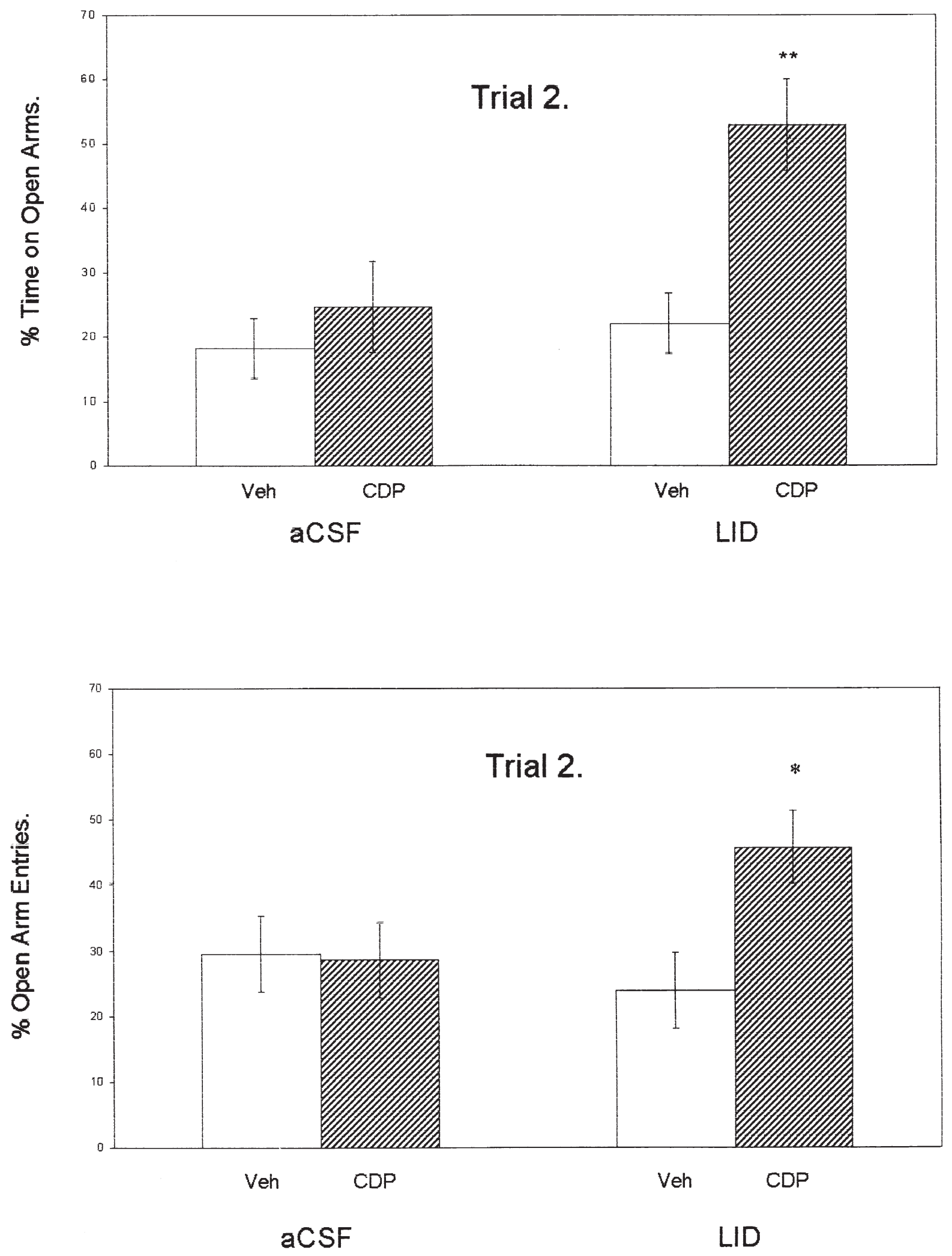

Figure 2. Mean $( \pm S E M)$ percentage of time spent on the open arms and percentage of open-arm entries made on trial 2 in the plus-maze by rats tested after IP injection with distilled water (VEH) or chlordiazepoxide (CDP, $2.5 \mathrm{mg} / \mathrm{kg}) . \mathrm{All}$ rats were undrugged on trial 1, but immediately after this trial, they had received vehicle (aCSF) or lidocaine (LID) injections into the basolateral nucleus of the amygdala. ${ }^{* *} p<.01,{ }^{*} p<.05$ compared with VEH group, Duncan's test after ANOVA. 

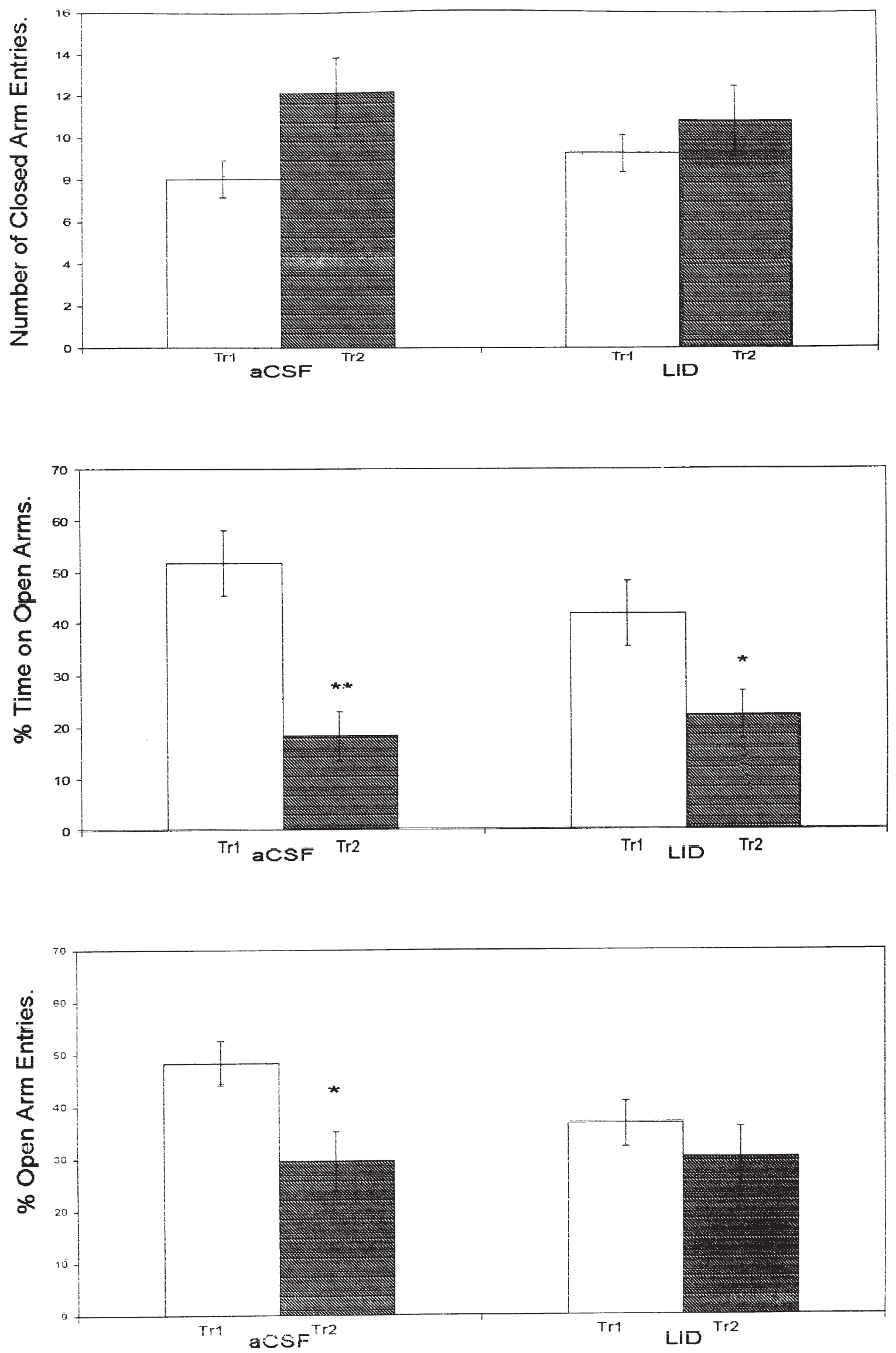

Figure 3. Mean $( \pm$ SEM) number of closed-arm entries, percentage of time spent on open arms, and percentage of openarm entries made by rats tested undrugged on trial $1(\operatorname{Tr} 1)$ and trial 2 ( $\operatorname{Tr} 2)$ in the plus-maze. Rats had received vehicle injections (ACSF) or lidocaine (LID) immediately after trial 1. ${ }^{*} p<.05,{ }^{* *} p<.01$ compared with trial 1, Duncan's test after ANOVA. 
habituation, because, in fact, there was a slight increase in closed-arm entries from trial 1 to 2 in the rats tested undrugged, see top panel, Figure 3 . These results suggest that the emotional memory of trial 1 , which led to an insensitivity to chlordiazepoxide on trial 2, was prevented by the lidocaine infusion.

It is possible to obtain an additional indication of the rat's prior exposure to the plus-maze from the betweentrial change in the scores of undrugged animals. Rather than habituating to the fear evoked by the open arms, rats tested undrugged on both trials show a betweentrial decrease in the percentage of time they spent on the open arms and the percentage of open-arm entries (Fernandes and File 1996). This was the pattern shown by the rats receiving aCSF after trial 1 and vehicle on trial 2, with significant decreases in both measures, see Figure 3 . The rats receiving a lidocaine injection after trial 1 and vehicle on trial 2, showed a significant between-trial decrease in the percentage of time spent on the open arms, but the decrease in percentage of openarm entries did not reach significance, see Figure 3.

\section{DISCUSSION}

The strategy employed in this experiment was to determine whether a reversible lesion of the basolateral nucleus of the amygdala could prevent consolidation of the emotional learning acquired during trial 1 in the elevated plus-maze. This was exactly the pattern that was found, with the rats that had received a lidocaine infusion immediately after trial 1 , showing a clear anxiolytic response to chlordiazepoxide on trial 2; that is, they behaved in the same way as rats that were naive to the plus-maze. This was in contrast to the animals that had received the control infusion of aCSF after trial 1 and who displayed the usual insensitivity to chlordiazepoxide on trial 2. This lack of sensitivity to chlordiazepoxide is unlikely to be attributable to a change in baseline responses on trial 2, because it is easier to detect a anxiolytic effect when the percentage of time spent on the open arms by undrugged animals is low. It has also been suggested (Dawson et al. 1994) that the decreased sensitivity to benzodiazepines on trial 2 is the result of locomotor habituation. This clearly was not the case in the present study, because the measure of locomotor activity, closed-arm entries, showed a slight increase. Decreased locomotor activity was also excluded as an explanation for benzodiazepine insensitivity in a previous study, using several different measures of locomotor activity (Gonzalez and File 1997). The effects of lidocaine administration in the amygdala resembled those seen after exposing rats to trial 1 in the plus-maze after an amnesic dose $(75 \mathrm{mg} / \mathrm{kg})$ of chlordiazepoxide (File et al. 1990).

Although the lidocaine infusion blocked the forma- tion of the specific phobia, it did not totally block the memory of trial 1 exposure. The rats tested undrugged on both trials showed a between-trial decrease in the percentage of time spent on the open arms, which was significant both for those that had received the lidocaine infusion after trial 1 and for those that had received the aCSF infusion. However, only the latter group showed a significant between-trial decrease in the percentage of open-arm entries, see Figure 3. It is not clear how the between-trial decreases in scores should be interpreted and whether they are an indication of greater anxiety on trial 2. However, it is clear that, whereas the basolateral nucleus is essential for the consolidation of information that changes the nature of the anxiety experienced on trial 2, it is not essential for consolidation of other information that leads to response suppression on trial 2. This pattern of results is similar to those of Killcross et al. (1997), who found that lesions of the basolateral nucleus did not impair all measures of aversive conditioning and, for example, impaired avoidance of a conditioned aversive stimulus, but did not impair the response suppression that it caused.

Our results from the elevated plus-maze confirm the suggestion (Killcross et al. 1997) that the basolateral nucleus of the amygdala plays a crucial role in active avoidance and conflict behavior and extends the previous findings based on lever pressing in an operant task to a situation where the response on trial 1 is unconditioned. Importantly, it also extends the findings to a situation in which the nature of the learning renders the animals insensitive to the anxiolytic effects of benzodiazepines in a subsequent plus-maze test. The design that we used allows us to identify the role of the basolateral nucleus as one crucial to the consolidation of information from trial 1; however, we cannot exclude the possibility that the nucleus also plays roles in acquisition and retrieval.

On the basis of previous studies (Parent and McGaugh 1994; Killcross et al. 1997) it would seem likely that these would be less crucial. This study did not address the role of the central nucleus of the amygdala because of previous findings (Killcross et al. 1997) that it was involved in automatic, reflexive responses, rather than choice behavior. It is unlikely that our results are attributable to the action of lidocaine in the central nucleus, because we were able to make accurate localization of our injection sites, because the acetylcholinesterase stain allowed clear visualization of the border of the basolateral nucleus, which is not possible with the thionin stain used in previous studies (Parent and McGaugh, 1994). Second, we used a very small volume of injection $(0.2 \mu \mathrm{l})$, and even with a larger volume $(0.5 \mu \mathrm{l})$, Pesold and Treit (1994) were able to demonstrate different effects of benzodiazepines following injections into the basolateral and central nuclei. 
Our results, in combination with those of Killcross et al. (1997) and Parent and McGaugh (1994), suggest that the basolateral nucleus plays a key role in the consolidation of information that leads to phobic avoidance. The fact that the specific phobia, fear of heights, is rapidly acquired during initial exposure to the plus-maze suggests that there is a genetic predisposition to acquire this fear. Conditioned fears based on punishment have not proved useful animal models of phobia (Klein 1981), but this rapidly acquired fear of heights using a naturalistic test situation may prove more useful. Very little in known about the neurobiology underlying specific phobias, and they are, in general, resistant to drug therapy (Marks 1987; Tyrer 1989).

The most important question, from the clinical point of view, is which drug treatments might prove effective in reversing specific phobias. At present, the most effective treatments are behavioral (Marks 1987), and these are time-consuming and limited by the available therapists. To establish possible therapies, it will be necessary to identify the brain regions crucial for the retrieval of the emotional information underlying a phobia and the neurotransmitter pathways and receptors involved. Recent positron emission tomography (PET) studies of snake (Fredrikson et al. 1993) and spider phobics (Fredrikson et al. 1995) confirm that the amygdala is not activated during the elicitation of a phobic response, but show increased activation of the secondary visual cortex and reduced activation of the hippocampus, prefrontal, and cingulate cortex. The PET studies also confirm that the phobic response and pattern of brain activation is not changed by diazepam (Fredrikson et al. 1995). These PET studies should provide a useful guide for future neuroanatomical and neuropharmacological studies using our animal model.

\section{ACKNOWLEDGMENT}

These experiments were supported by a grant from the Special Trustees of Guy's Hospital. We are grateful to Dr. Abdel Ouagazzal for assistance with surgery.

\section{REFERENCES}

Davis M (1992): The role of the amygdala in fear and anxiety. Ann Rev Neurosci 15:353-375

Dawson GR, Crawford SP, Stanhope KJ, Iversen SD, Tricklebank MD (1994): One-trial tolerance to the effects of chlordiazepoxide on the elevated plus-maze may be due to locomotor habituation, not repeated drug exposure. Psychopharmacology 113:570-572

Everitt BJ, Robbins TW (1992): Amygdala-ventral striatal interaction and reward related processes. In Aggleton JP (ed), The Amygdala: Neurobiological Aspects of Emo- tion, Memory, and Mental Dysfunction. New York, Wiley-Liss, pp 401-429

Fernandes C, File SE (1996): The influence of open-arm ledges and maze experience in the elevated plus-maze. Pharmacol Biochem Behav 54:31-40

File SE (1990): One-trial tolerance to the anxiolytic effects of chlordiazepoxide in the plus-maze. Psychopharmacology 100:281-282

File SE (1991): The biological basis of anxiety. In Meltzer HY, Nerozzi D (eds), Current Practices and Future Developments in the Pharmacotherapy of Mental Disorders. Amsterdam, Ex Medica, pp 159-165

File SE (1993): The interplay of learning and anxiety in the elevated plus-maze. Behav Brain Res 58:199-202

File SE, Gonzalez LE (1996): Anxiolytic effects in the plusmaze of 5- $\mathrm{HT}_{1 \mathrm{~A}}$ receptor ligands in dorsal raphe and ventral hippocampus. Pharmacol Biochem Behav 54:123-128

File SE, Zangrossi H (1993): “One-trial tolerance” to the anxiolytic actions of benzodiazepines in the elevated plusmaze, or the development of a phobic state? Psychopharmacology 110:240-244

File SE, Andrews N, Hogg S (1996): New developments in animal tests of anxiety. In Westenberg HGM, Den Boer JA, Murphy DL (eds), Advances in the Neurobiology of Anxiety Disorders. Chichester, UK, Wiley, pp 61-79

File SE, Mabbutt PS, Hitchcott P (1990): Characterization of the phenomenon of "one-trial tolerance" to the anxiolytic effect of chlordiazepoxide in the elevated plusmaze. Psychopharmacology (Berlin) 102:98-101

File SE, Zangrossi H, Viana M, Graeff FG (1993): Trial 2 in the elevated plus-maze: A different form of fear? Psychopharmacology 111:494

Fredrikson M, Wik G, Greitz T, Eriksson L, Stone-Elander S (1993): Regional cerebral blood flow during experimental phobic fear. Psychophysiology 30:127-131

Fredrikson M, Wik G, Annas P, Ericson K, Stone-Elander S (1995): Functional neuroanatomy of visually elicited simple phobic fear: Additional data and theoretical analysis. Psychophysiology 32:43-48

Gonzalez LE, File SE (1997): A five minute experience in the elevated plus-maze alters the state of the benzodiazepine receptor in the dorsal raphe nucleus. J Neuroscience 17:1505-1511

Killcross S, Robbins TW, Everitt BJ (1997): Different types of fear-conditioned behavior mediated by separate nuclei within amygdala. Nature 388:377-380

Klein DF (1981): Anxiety reconceptualized. In Klein DF, Rabkin J (eds), Anxiety: New Research and Changing Concepts. New York, Raven Press, pp 235-263

LeDoux J (1996): The Emotional Brain. New York, Simon \& Schuster

McGaugh JL (1995): Emotional activation, neuromodulatory systems, and memory. In Schacter DL, Coyle JT, Mesulam M-M, Sullivan LE (eds), Memory Distortion: How Minds, Brains, and Societies Reconstruct the Past. Cambridge, MA, Harvard University Press, pp 255-273

McGaugh JL, Cahill L, Roozendall B (1996): Involvement of the amygdala in memory storage: Interaction with other brain systems. Proc Natl Acad Sci USA 93:13508-13514 
Marks IM (1987): Fears, Phobias, and Rituals. New York, Oxford University Press

Parent MB, McGaugh JL (1994): Post-training infusion of lidocaine into the amygdala basolateral complex impairs retention of inhibitory avoidance training. Brain Res 661:97-103

Paxinos G, Watson CH (1986): The Rat Brain in Stereotaxic Coordinates. London, Academic

Pellow S, Chopin P, File SE, Briley M (1985): Validation of open- closed-arm entries in an elevated plus-maze as a measure of anxiety in the rat. J Neurosci Meth 14:149167

Pesold C, Treit D (1994): The septum and amygdala differentially mediate the anxioltyic effects of benzodiazepines. Brain Re 638:295-301

Pesold C, Treit D (1995): The central and basolateral amygdala differentially mediate the anxiolytic effects of benzodiazepines. Brain Res 671:213-221
Rodgers RJ, Johnson NJT (1995): Factor analysis of spatiotemporal and ethological measures in the murine elevated plus-maze test of anxiety. Pharmacol Biochem Behav 52:297-303

Rodgers RJ, Shepherd JK (1993): Influence of prior maze experience on behavior and response to diazepam in the elevated plus-maze and light/dark tests of anxiety in mice. Psychopharmacology 113:237-242

Rodgers RJ, Lee C, Shepherd JK (1992): Effects of diazepam on behavioral and antinociceptive responses to the elevated plus-maze in male mice depend upon treatment regimen and prior maze experience. Psychopharmacology 106:102-110

Treit D, Menard J, Royan C (1993): Anxiogenic stimuli in the elevated plus-maze. Pharmacol Biochem Behav 44:463469

Tyrer P (1989): Choice of treatment in anxiety. In Tyrer P (ed), Psychopharmacology of Anxiety. Oxford, UK, Oxford Medical Publications, pp 254-282 